\title{
Measurements of Nucleon Structure via Proton-Induced Drell-Yan Process at FNAL SeaQuest
}

\author{
Kei Nagai ${ }^{* \dagger}$ \\ Institute of Physics, Academia Sinica, Taiwan \\ E-mail: Enagaidgate.sinica.edu.tw
}

\begin{abstract}
SeaQuest at FNAL is a fixed-target experiment to measure the Drell-Yan process in $p+p$ and $p+A$, using the $120-\mathrm{GeV}$ proton beam and targets of liquid hydrogen, liquid deuterium, carbon, iron and tungsten. SeaQuest aims at precisely measuring the flavor asymmetry of light-antiquark distributions $(\bar{d}(x) / \bar{u}(x))$ at large $x$ in the nucleon. It also measures the angular distribution of the Drell-Yan process in $p+p$ and $p+d$ in order to investigate the Lam-Tung relation and further the Boer-Mulders distribution function. It also utilizes the Drell-Yan process in $p+A$ for measuring nuclear effects, including the nuclear dependence of sea quark distributions and the parton energy loss in cold nuclear matter. Here, the results of $\bar{d} / \bar{u}$ are shown.
\end{abstract}

XXVI International Workshop on Deep-Inelastic Scattering and Related Subjects (DIS2018) 16-20 April 2018

Kobe, Japan

* Speaker.

${ }^{\dagger}$ On behalf of the SeaQuest Collaboration 


\section{Introduction}

The Drell-Yan process [四] is a reaction where an antiquark in a hadron and a quark in another hadron annihilate and then decay into a lepton pair via a virtual photon in a high energy hadronhadron scattering $\left(q+\bar{q} \rightarrow \gamma^{*} \rightarrow l+\bar{l}\right)$. The differential cross section of proton-proton Drell-Yan process at leading order is described as

$$
\frac{d^{2} \sigma}{d x_{\text {beam }} d x_{\text {target }}}=\frac{4 \pi \alpha^{2}}{9 x_{\text {beam }} x_{\text {target }}} \frac{1}{s} \sum_{i=u, d, s, \ldots} e_{i}^{2}\left[q_{i}\left(x_{\text {beam }}\right) \bar{q}_{i}\left(x_{\text {target }}\right)+\bar{q}_{i}\left(x_{\text {beam }}\right) q_{i}\left(x_{\text {target }}\right)\right] .
$$

The last term of Eq. (ㅁ) vanishes in forward detection $\left(x_{\text {beam }} \gg x_{\text {target }}\right)$ because the PDFs of antiquarks at large $x$ are small. It allows us to access the antiquarks in target protons and quarks in beam protons. The Drell-Yan process is a unique probe for antiquarks. Another remarkable property of the Drell-Yan process is that there is no strong interaction in the final state. It makes the initial state effect stand out in the measurement.

Therefore, SeaQuest aims at measuring the property of antiquarks and the nuclear effects via the Drell-Yan process.

\section{SeaQuest Experiment}

The SeaQuest experiment is performed at Fermi National Accelerator Laboratory (FNAL) and is collaborated by U.S., Japan, and Taiwan institutes. The main subjects are 1) antiquark flavor asymmetry, 2) parton energy loss, and 3) angular distribution. They are extracted by measuring the dimuons in the final state of Drell-Yan process. The SeaQuest spectrometer was designed to measure the Drell-Yan dimuons momenta.

Figure $\mathbb{U}$ shows the SeaQuest spectrometer. The incident proton beam comes from the lefthand side of the figure and collides with targets. The proton beam is provided by the Fermilab Main Injector. The energy is $120 \mathrm{GeV}$. The targets are liquid hydrogen $\left(\mathrm{LH}_{2}\right)$, liquid deuterium $\left(\mathrm{LD}_{2}\right)$, iron, carbon, and tungsten. The empty flask which is the same as the container used for liquid targets is also located at the target position for the reference. The liquid targets are for the antiquark flavor asymmetry and angular distribution studies, and solid targets are for the nuclear effects. The dimuons created at the target area pass through the focusing magnet. The magnet focuses the dimuons which are proper for the experiment on the spectrometer. Four tracking stations detect the proper dimuons. A station is a group of detectors: each of St. 1, 2 and 3 consists of a hodoscope array and a drift chamber, and St. 4 consists of a hodoscope array and proportional tubes. The muon momenta are determined by $\mathrm{KTeV}$ magnet located between St. 1 and St. 2. A hadron absorber is located between St. 3 and St. 4 for the muon identification.

SeaQuest finished the detector construction in 2011 and started the physics data taking in 2013. After four periods, SeaQuest finished the physics data runs in 2017. The recorded number of protons on targets is $1.4 \times 10^{18}$.

Figure $\square$ shows the invariant mass spectrum of reconstructed dimuons. The amount of data is approximately $5 \%$ of final data set. It is fitted with four components: Drell-Yan (red line), $J / \psi$, and $\psi^{\prime}$ (magenta lines) dimuons estimated with Monte Carlo simulation and random background (black line) estimated with real data by event mixing method. The experimental data (black points) 


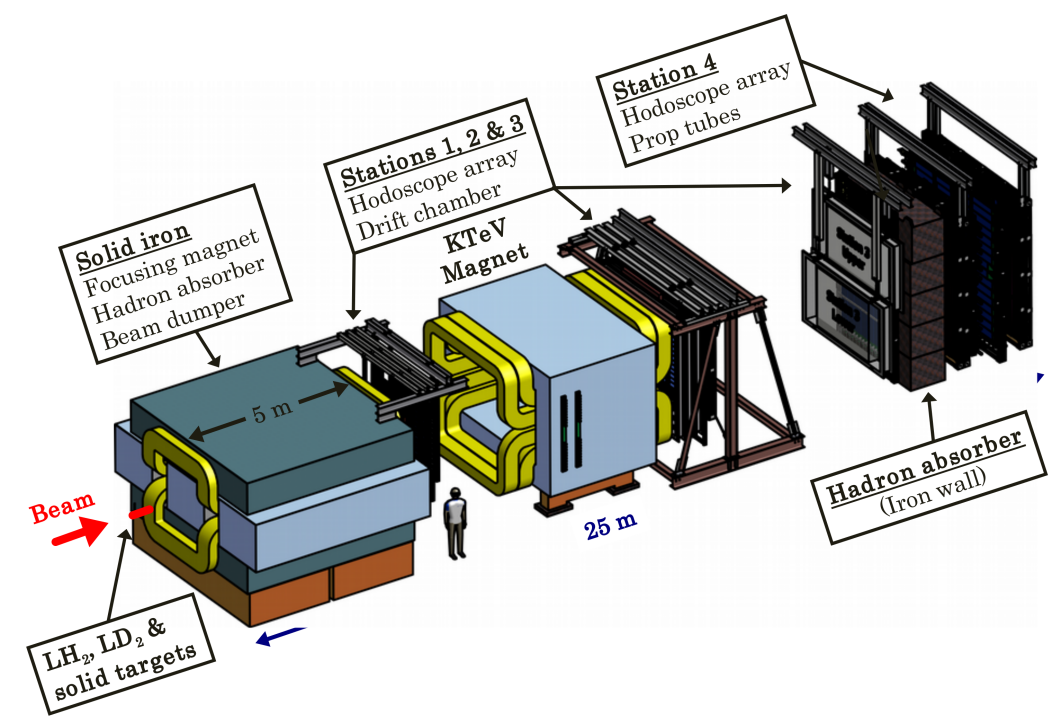

Figure 1: SeaQuest spectrometer. It was designed for measuring the momenta of dimuons originated from the Drell-Yan process. The spectrometer consists of targets, two hadron absorber, two dipole magnets, and four tracking stations.

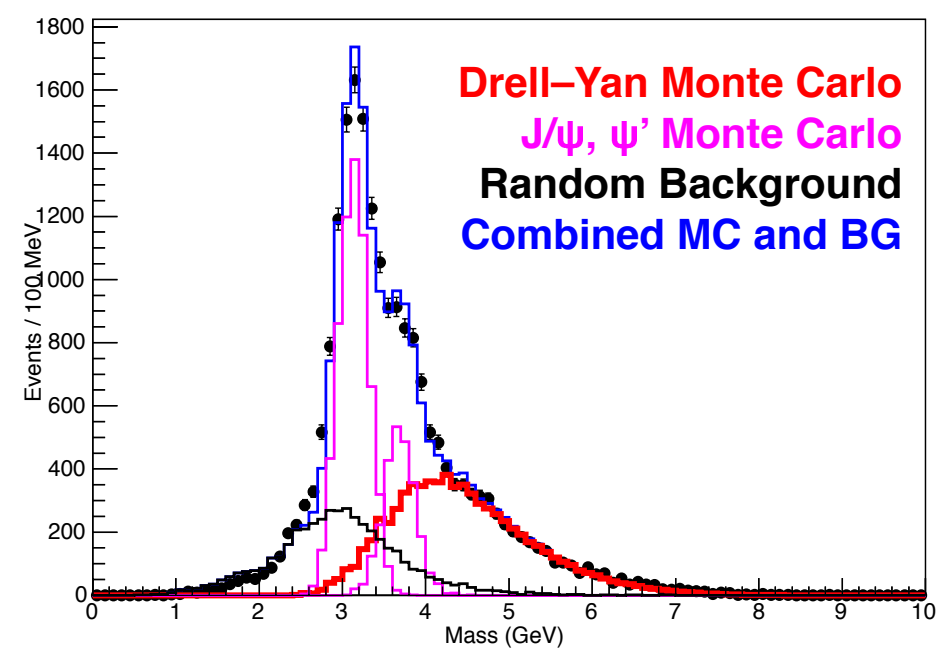

Figure 2: The invariant mass spectrum of reconstructed dimuons. The distributions of Drell-Yan (red), $J / \psi$, and $\psi^{\prime}$ (magenta) are estimated by Monte Carlo simulation. The random background is estimated with real data by event mixing method. The blue line is the fit of the real data (black points) with those four components. 
are well fitted with the blue line which describes the sum of the four components. The dimuons with invariant mass $>4.2 \mathrm{GeV}$ are used for the physics analysis because the Drell-Yan dimuons are dominated in that region.

The analyses of parton energy loss and angular distribution are too preliminary to be shown. Here, the preliminary result of the antiquark flavor asymmetry is shown.

\section{Antiquark Flavor Asymmetry $\bar{d} / \bar{u}$}

The antiquarks in the proton are the sea quarks and are generated from the gluon splitting $(g \rightarrow q+\bar{q})$. The probabilities that the gluon splits into $u \bar{u}$ pair and $d \bar{d}$ pair are the same because the coupling constant of the strong interaction is independent of the quark flavor and the current quark masses are more or less equal for $u$ and $d$ quarks. It is expected that the amount of $\bar{d}$ in the proton is the same as that of $\bar{u}$ (flavor symmetry).

The NMC experiment at CERN is the first experiment to test the flavor symmetry using the deep inelastic muon scattering [D]. The NMC experiment found that the total amount of $\bar{d}$ in the proton is larger than that of $\bar{u}\left(\int_{0}^{1} \bar{d}(x) d x>\int_{0}^{1} \bar{u}(x) d x\right)$. The NA51 experiment at CERN and E866 experiment at FNAL measured the antiquark PDFs ratio of $\bar{d}(x)$ to $\bar{u}(x)$ as a function of $x$ using the Drell-Yan process [ [B], 团]. They found $\bar{d}(x) / \bar{u}(x)>1.0$ at each $x$ region. In particular, a $70 \%$ asymmetry at maximum has been found at $x \sim 0.2$. These results agreed with the results from the NMC experiment. However, E866 experiment showed $\bar{d} / \bar{u}<1.0$ at $x \sim 0.3$, but with large statistical uncertainty.

Based on the NMC, NA51, and E866 experiments results, many models were proposed to understand the behavior of $\bar{d}>\bar{u}$ : Pauli blocking [可], statistical model [罒, meson cloud model [प], and so on. Some models succeeded in reproducing the tendency of $\bar{d} / \bar{u}>1.0$. However, no model can reproduce the tendency of $\bar{d} / \bar{u}<1.0$ which was shown by the E866 experiment. It is very interesting also for the theoretical study if $\bar{d} / \bar{u}<1.0$ or not in high $x$ region. Thus, the conclusive result at high $x$ is very important to understand the proton structure. The SeaQuest experiment measures $\bar{d} / \bar{u}$ precisely at high and wide $x$ range $(0.15<x<0.45)$ including the intriguing region in E866.

The relation between the antiquark flavor asymmetry and the Drell-Yan cross section ratio of proton-deuteron to proton-proton is derived from Eq. (메):

$$
\frac{\sigma_{p d}(x)}{2 \sigma_{p p}(x)} \approx \frac{1}{2}\left[1+\frac{\bar{d}(x)}{\bar{u}(x)}\right]
$$

under $x_{\text {beam }} \gg x_{\text {target }}$. SeaQuest measures the Drell-Yan cross section ratio as a function of $x$ to extract $\bar{d} / \bar{u}$. The cross section ratio is obtained by

$$
\frac{\sigma_{p d}}{2 \sigma_{p p}}=\frac{1}{2}\left(\frac{N_{D} \cdot C_{D}}{P_{D}}\right) /\left(\frac{N_{H} \cdot C_{H}}{P_{H}}\right),
$$

where subscripts $D$ and $H$ denote the $\mathrm{LD}_{2}$ and $\mathrm{LH}_{2}$ targets, respectively, $N$ is the number of measured dimuons, $C$ is the correction factors including the background correction and reconstruction efficiency correction, and $P$ is the product of the numbers of nucleons in beam and targets. Figure $B$ shows the preliminary result of the cross section ratio from SeaQuest FY 2015 data. Blue line 


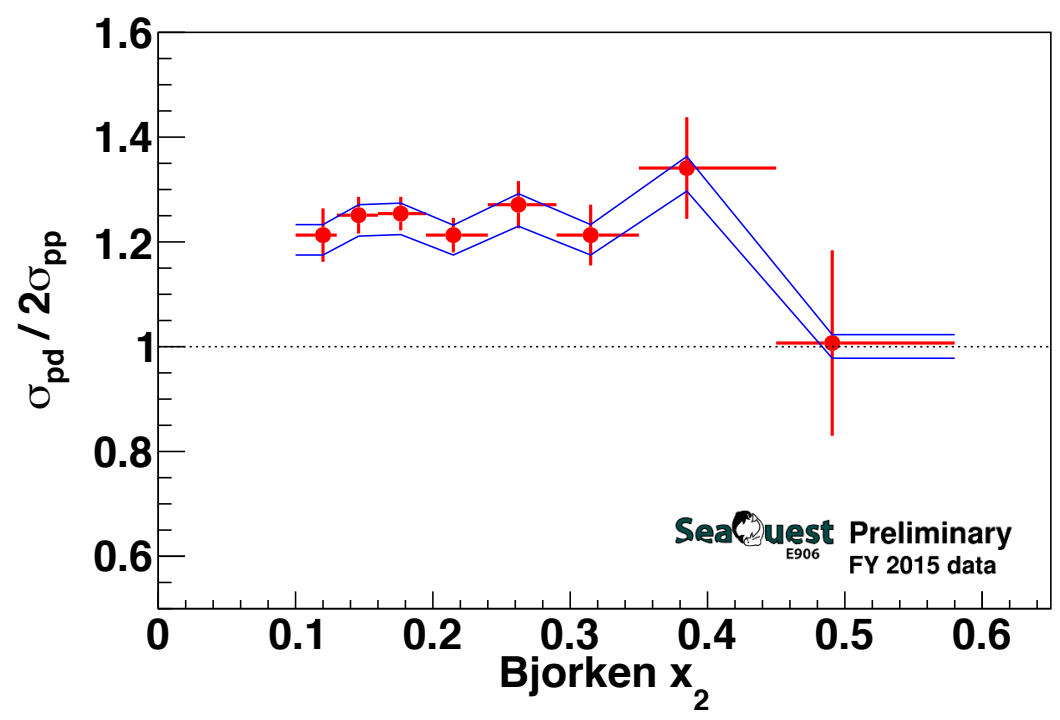

Figure 3: Preliminary result of cross section ratio of proton-deuterium to proton-proton Drell-Yan processes with FY 2015 data. Blue line shows the systematic uncertainty.

shows the systematic uncertainty. The cross section ratios in $0.1<x<0.58$ were extracted.

The cross section ratio is converted into $\bar{d} / \bar{u}$ using Eq. (ㅁ. it is an approximated formula and is not very accurate. The conversion is performed with the iterative analysis. The cross section ratio is predicted using the CT10LO PDF [ $[\mathbb{8}](u, d, s, c, \bar{d}+\bar{u})$ and the estimate of $\bar{d} / \bar{u}$. The estimate of $\bar{d} / \bar{u}$ is set 1.0 at the first iteration. If the difference between predicted and measured cross section ratios is not small enough, the estimate of $\bar{d} / \bar{u}$ is updated based on the difference of cross section ratios. This procedure is repeated until the difference becomes small enough. The estimate of $\bar{d} / \bar{u}$ after this iterative analysis is adopted as the result.

Figure $⿴$ shows the preliminary result of $\bar{d}(x) / \bar{u}(x)$ in $0.1<x<0.58$ with FY 2015 data drawn with the results from NA51 and E866. The systematic uncertainty includes hydrogen contamination in $\mathrm{LD}_{2}$, random background, hit-rate dependence of reconstruction efficiency, and uncertainty from CT10LO PDF. We extracted $\bar{d}(x) / \bar{u}(x)$ in $0.1<x<0.58$, which is the wider $x$-coverage compared to E866. In $0.1<x<0.24$, the results are consistent with NA51 and E866 within statistical uncertainty. On the other hand, the results at large $x$ are higher than those from E866. This discrepancy is under investigation. The difference of $Q^{2}$ between SeaQuest and E866 has already been studied and does not affect much. Possible causes are the nuclear effects on deuterium, the hit-rate dependence, and the background subtraction.

For the final results, we are improving $\mathrm{S} / \mathrm{N}$ ratio and hit-rate dependence correction, estimating the nuclear effect on $\mathrm{LD}_{2}$, and extracting $\bar{d} / \bar{u}$ in the next-to-leading order [Q, प, प]]. The statistics will also increase. The preliminary results are based on approximately $40 \%$ of obtained protons on targets. The latest data taking was performed with the larger acceptance and improved trigger. It suggests that the analyzable data rate will be much more than that in FY 2015 data. We will provide more precise results soon. 


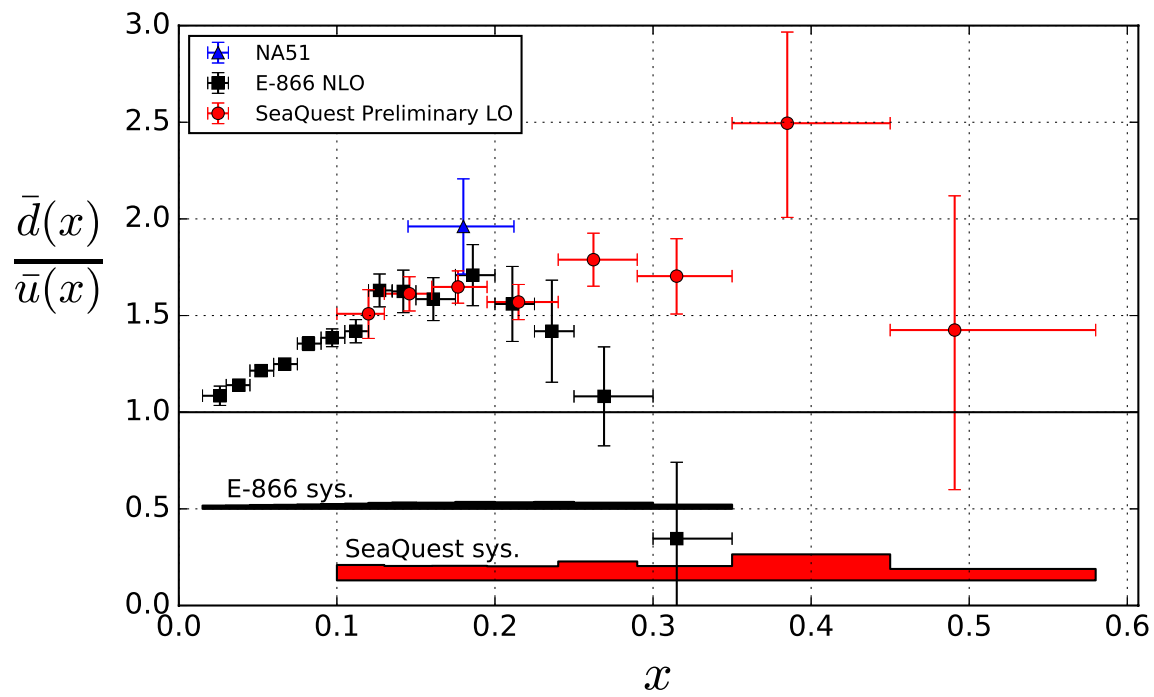

Figure 4: Preliminary result of antiquark flavor asymmetry $\bar{d}(x) / \bar{u}(x)$ with FY 2015 data (red) drawn with the results from NA51 (blue) and E866 (black). The red band shows the systematic uncertainty of the SeaQuest result.

\section{References}

[1] S. D. Drell and T. M. Yan, Massive Lepton-Pair Production in Hadron-Hadron Collisions at High Energies, PRL 25 (1970) 316.

[2] M. Arneodo et al., Reevaluation of the Gottfried sum, PRD 50 (1994) R1.

[3] A. Baldit et al., Study of the isospin symmetry breaking in the light quark sea of the nucleon from the Drell-Yan process, PLB 332 (1994) 244.

[4] R. S. Towell et al., Improved measurement of the $\bar{d} / \bar{u}$ asymmetry in the nucleon sea, PRD 64 (2001) 052002.

[5] R. D. Field, and R. P. Feynman, Quark elastic scattering as a source of high-transverse-momentum mesons, PRD 15 (1977) 2590.

[6] E. Basso, C. Bourrely, R. Pasechnik and J. Soffer, The Drell-Yan process as a testing ground for parton distributions up to LHC, NPA 948 (2016) 63.

[7] J. C. Peng et al., $\bar{d} / \bar{u}$ asymmetry and the origin of the nucleon sea, PRD 58 (1998) 092004.

[8] H. Lai et al., New parton distributions for collider physics, PRD 82 (2010) 074024.

[9] S. Miyasaka, Probing Flavor Asymmetry of Anti-quarks in the Proton by Drell-Yan Experiment SeaQuest, Ph.D. thesis, Tokyo Tech, 2016.

[10] K. Nagai, Recent Measurement of Flavor Asymmetry of Antiquarks in the Proton by Drell-Yan Experiment SeaQuest at Fermilab, Ph.D. thesis, Tokyo Tech, 2017.

[11] B. Kerns, A measurement of the $x$ dependent sea flavor asymmetry at SeaQuest, Ph.D. thesis, UIUC, 2017. 